\title{
Perforation of small intestinal metastasis of lung adenocarcinoma treated with pembrolizumab: a case report
}

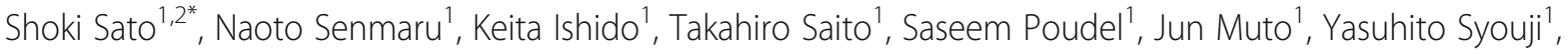
Ryunosuke Hase ${ }^{1}$ and Satoshi Hirano ${ }^{2}$

\begin{abstract}
Background: Pembrolizumab is an immune checkpoint inhibitor and is an anti-human programmed cell death-1 (PD-1) monoclonal antibody. Pembrolizumab is used for non-small cell lung carcinoma with high programmed cell death ligand-1 (PD-L1) expression. It has been found that better overall survival can be obtained using pembrolizumab compared to the existing chemotherapy. We report a case of perforation of small intestinal metastasis after pembrolizumab treatment.

Case presentation: A 62-year-old man was treated by pembrolizumab for PD-L1 highly expressed lung adenocarcinoma, with multiple metastasis (small intestinal, lymph nodes, and bone). The treatment was stopped owing to drug-induced pneumonitis. One month after drug withdrawal, the patient visited the emergency department of our hospital with the complaint of severe stomachache. He had a rigid abdomen and generalized tenderness, and computed tomography scans showed free air within the abdomen. We diagnosed bowel perforation and performed emergency surgery. Surgical findings revealed multiple small intestine metastasis and mesenteric lymph node metastasis. Perforation was found in the metastatic site in the jejunum located around $40 \mathrm{~cm}$ anal to Treitz's ligament. This perforated part was resected, and functional end-to-end anastomosis was performed using linear staplers. The post-operative course was uneventful. Pathological examination revealed lung adenocarcinoma metastasis at the perforation site, and the effectiveness of pembrolizumab was grade $1 \mathrm{~b}$ (Japanese Classification of the Colorectal Carcinoma, seventh edition).
\end{abstract}

Conclusions: This is the first report of perforation of small intestinal metastasis of lung adenocarcinoma after pembrolizumab treatment. Because pembrolizumab causes some side effects, particularly autoimmune side effects, careful attention during treatment is warranted.

Keywords: Pembrolizumab, Small intestinal perforation, Non-small cell lung carcinoma metastasis

\section{Background}

Pembrolizumab, an immune checkpoint inhibitor, is an anti-human programmed cell death-1 (PD-1) monoclonal antibody and used for the treatment of non-small cell lung carcinoma (NSCLC) and melanoma with high expression of programmed cell death ligand-1 (PD-L1) and high microsatellite instability (MSI) status solid cancer [1-3].

\footnotetext{
* Correspondence: a02m033@yahoo.co.jp

${ }^{1}$ Department of Surgery, Steel Memorial Muroran Hospital, 1-45,

Chiribetu-chou, Muroran, Hokkaido 050-0046, Japan

${ }^{2}$ Department of Gastroenterological Surgery II, Hokkaido University Faculty of

Medicine, N-15 W-7, Sapporo, Hokkaido 060-8638, Japan
}

Anti-PD-1 antibodies, including pembrolizumab, are reported to have not only general chemotherapy side effect, for example nausea, leukopenia, and more, but also characteristic autoimmune side effects like hypothyroidism, type 1 diabetes, hypopituitarism, colitis, and drug-induced pneumonitis due to excessive immune reaction $[4,5]$. However, intestinal perforation caused by this drug has rarely been reported. We report a case of perforation of small intestinal metastasis of lung adenocarcinoma after pembrolizumab treatment. 


\section{Case presentation}

A 62-year-old man was treated with pembrolizumab for right lung adenocarcinoma, which showed high PD-L1 expression (80\%), with multiple intestinal, lymph node, and bone metastases. The TNM classification for NSCLC was cT2N3M1c (OSS, LYM, PER, OTH), stage IVB (eighth edition). Tumor reduction was observed, but pembrolizumab was stopped after three courses owing to druginduced pneumonitis. Dexamethasone was used for the treatment of pneumonitis. One month after drug withdrawal, the patient was transported to the emergency department of our hospital with the complaint of severe stomachache. On physical examination, he had a rigid abdomen and generalized tenderness. His blood pressure was in the normal range $(110 / 82 \mathrm{mmHg})$, the heart rate was elevated but regular at 100 beats per minute, and the body temperature was elevated at $38.9^{\circ} \mathrm{C}$. The peripheral capillary oxygen saturation was $98 \%$ at room air. Laboratory evaluation showed a high inflammatory response with a white blood cell count of $18,200 / \mathrm{mm}^{3}$ and C-reactive protein level of $20.8 \mathrm{mg} / \mathrm{dL}$. CT examination showed abdominal free air and ascites with perforation of the

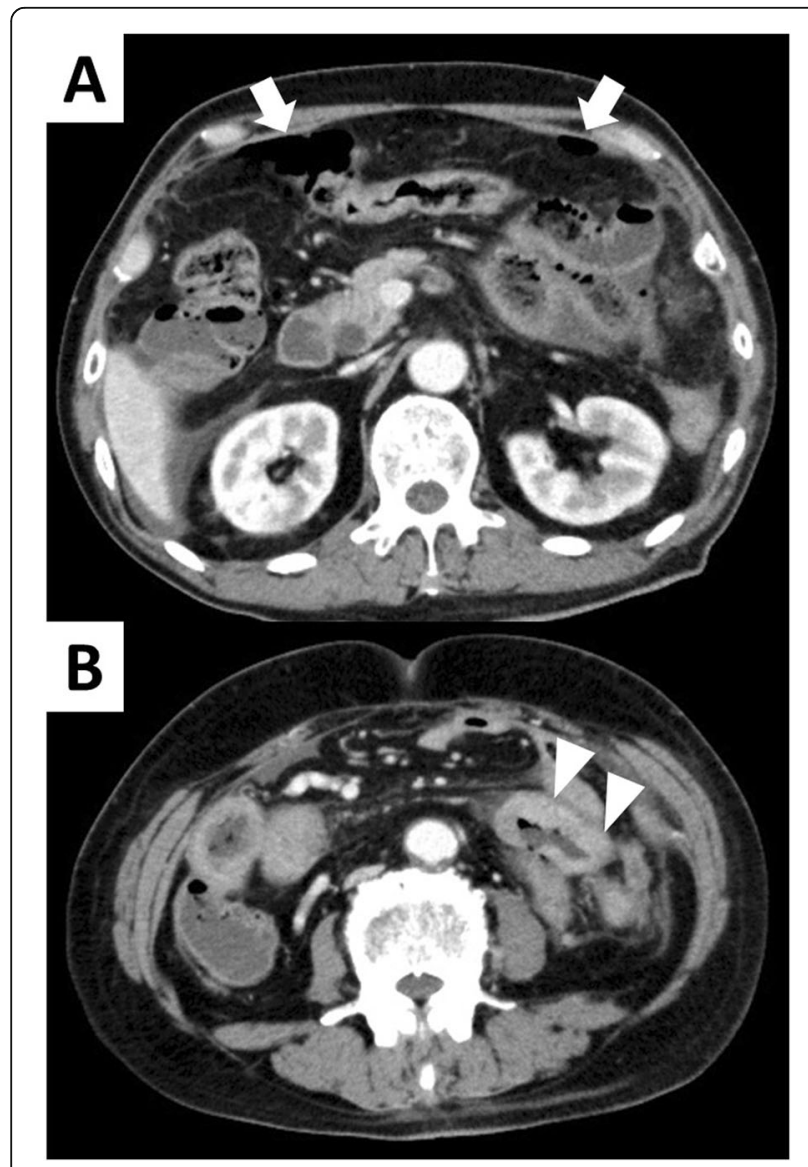

Fig. 1 a, b Abdominal enhanced CT pictures are shown. Arrows show abdominal free air, and triangles show the perforation site of small intestinal metastasis of lung adenocarcinoma existing lung adenocarcinoma metastasis (Fig. 1). We diagnosed bowel perforation with acute diffuse peritonitis. Emergency laparotomy was performed, and multiple small intestinal metastasis with mesenteric lymph node metastasis and ascites containing intestinal fluid were observed. The perforation site was located in the metastatic jejunum about $40 \mathrm{~cm}$ on the anal side from Treitz's ligament. We resected this part about $20 \mathrm{~cm}$ and anastomosed with functional end-to-end anastomosis. There was no complication after surgery, and he was discharged on postoperative day 15. Pathological examination indicated lung adenocarcinoma metastasis in the perforated intestine, and the metastasis was partly scarred owing to the effect of pembrolizumab (Fig. 2). Tumor cells in the perforation site had a high degree of degeneration and necrosis, and the pathological response for the efficacy of pembrolizumab was grade $1 \mathrm{~b}$ (Japanese Classification of the Colorectal Carcinoma, seventh edition) (Fig. 3). In the perforated part, the tumor cells were observed in all layers, but in the vicinity on the serosa side. Inflammatory change due to enteritis was not found in this site. Pembrolizumab was re-administrated about 1 month after discharge. To

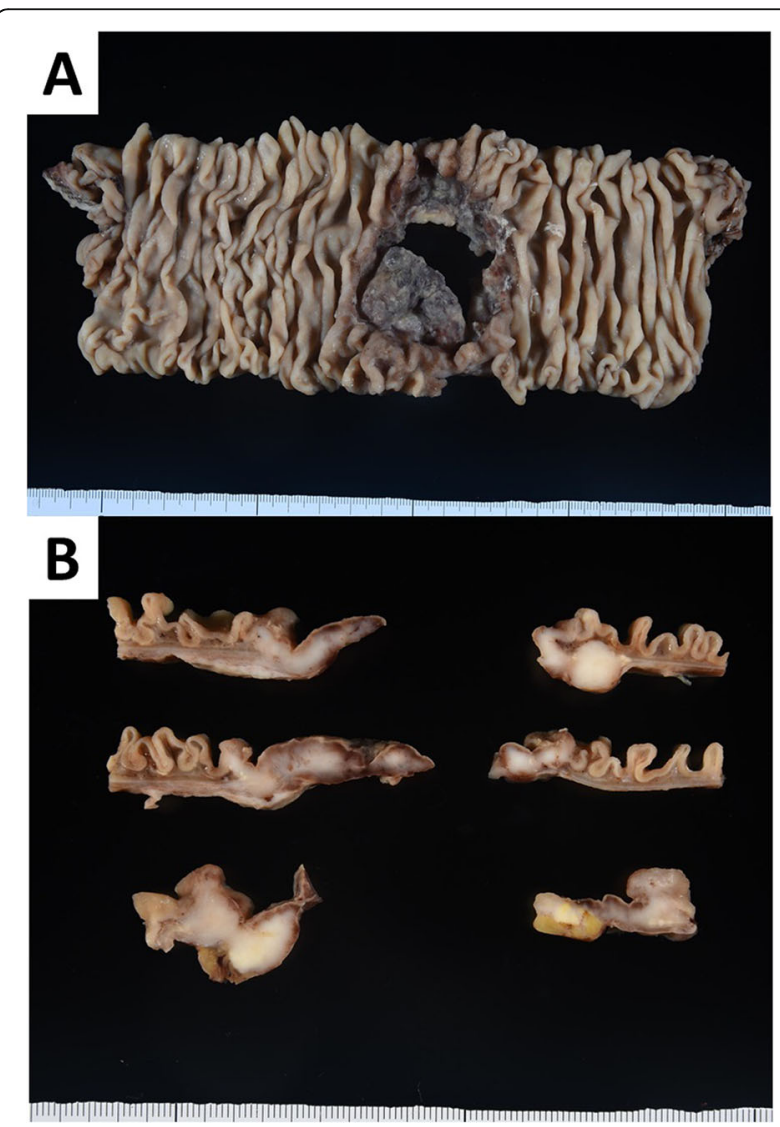

Fig. 2 The resected small intestine of perforation site with lung adenocarcinoma metastasis. a There was about $5 \times 4 \mathrm{~cm}$ perforation site with fibrotic change. $\mathbf{b}$ White tumors were found in the section of the small intestinal perforation site 


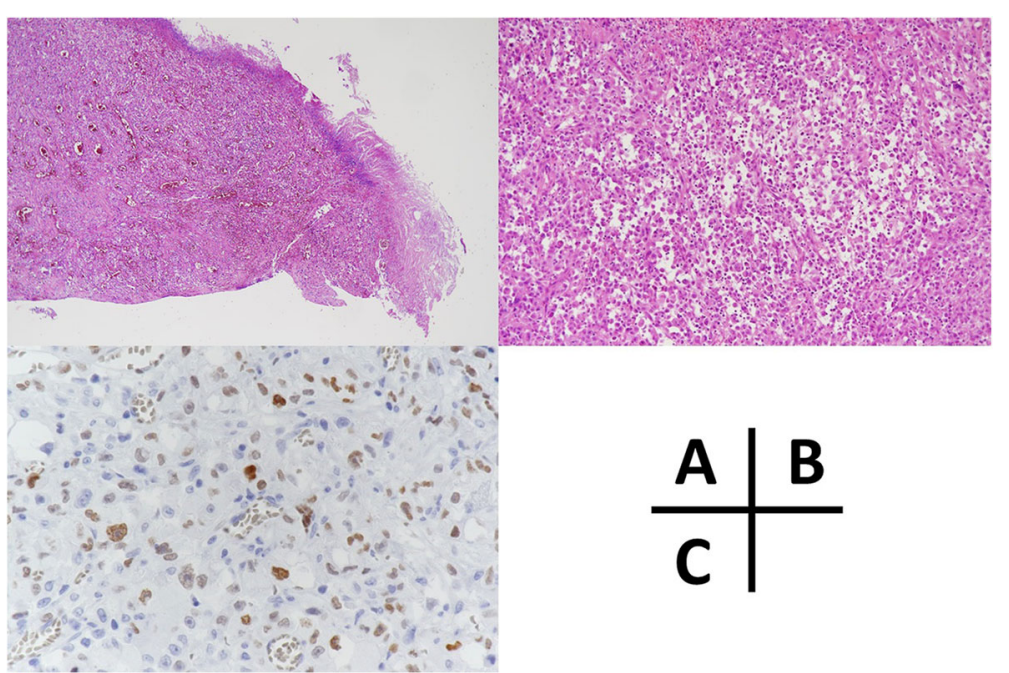

Fig. 3 Pathological examination and immunohistochemical stain for thyroid transcription factor 1 (TTF-1). a The right side of the picture was the perforated site, and tumor cells and fibrosis were observed throughout the whole layer $(\times 40)$. b There were tumor cells with high degree of degeneration and necrosis change in the perforation site $(\times 100)$. The pathological response for the efficacy of pembrolizumab was grade $1 \mathrm{~b}$ (Japanese Classification of the Colorectal Carcinoma, seventh edition). c The tumor cells showed TTF-1 expression in immunohistochemical stain

prevent drug-induced pneumonitis, dexamethasone was used daily.

\section{Discussion}

This is the first case report of intestinal perforation caused by the effect of pembrolizumab for NSCLC intestinal metastasis. One case of small intestinal perforation caused by metastasis of melanoma treated with pembrolizumab was reported in Italy [6]. In this case, multiple intestinal metastases were occurred, and some tumors had lined the entire intestinal wall in CT. Perforation cases of NSCLC intestinal metastasis treated with general chemotherapy were reported, and emergency surgery was done [7-9].

The interaction between PD-1 receptor with its ligands, PD-L1 and PD-L2, regulates the balance between T cell activation, immune tolerance, and immune-related tissue damage, and in tumor environment, this pathway is taken over to evade immune surveillance $[10,11]$. Pembrolizumab is a highly selective humanized immunoglobulin $\mathrm{G} 4 / \mathrm{K}$ monoclonal antibody designed to directly inhibit between PD-1 receptor and PD-L1/PD-L2 interaction by binding to PD-1 receptor. In KEYNOTE-024 trial, advanced NSCLC patient group treated with pembrolizumab had longer progression-free survival than those treated with chemotherapy, and the median progression-free survival was 10.3 months vs. 6.0 months, respectively $(P<0.001)[4]$.

Anti-PD-1 antibodies, including pembrolizumab, have many side effects ranging from leukopenia, nausea, and fatigue, which are commonly seen with other anti-cancer drugs, to side effects attributed to the autoimmune response to this drug like hypothyroidism, type 1 diabetes, hypopituitarism, colitis, and drug-induced pneumonitis $[4,5]$. The patient in our case also experienced druginduced pneumonitis, which was treated with dexamethasone. Hence, there was an increased risk of perioperative infection (surgical site infection and abdominal abscess) and anastomosis failure. Fortunately, there were no major complications or side effects due to excessive immune reaction. Generally, characteristic side effects of pembrolizumab occur immediately after administration. However, it is not uncommon to see the side effects after a few months [5]. Patients who have been treated with pembrolizumab should always consider autoimmune side effects, and it is necessary to treat these as soon as their onset is suspected.

\section{Conclusions}

This is the first report of perforation of NSCLC small intestinal metastasis after pembrolizumab treatment. It is necessary to perform perioperative management with the onset of autoimmune side effects characteristic of anti-PD-1 antibodies, including pembrolizumab.

\section{Abbreviation \\ CT: Computer tomography}

\section{Acknowledgements}

We thank Ms. Miri Fujita for reviewing the pathology slides and facilitating the use of the phytopathology.

\section{Authors' contributions}

SS and JM performed the operation. SS, PS, and JM managed the perioperative course. SS wrote the manuscript. PS supported in the writing of the manuscript. YS, RH, NS, and SH supervised the writing of the manuscript. All authors read and approved the final manuscript. 
Funding

This research received no specific grant from any funding agency.

\section{Availability of data and materials}

The datasets supporting the conclusions of this article are included within this article.

\section{Ethics approval and consent to participate}

This study was conducted in accordance with the ethical standards of our hospital.

\section{Consent for publication}

Informed consent for publication of the patient's clinical details and images was obtained from the wife.

\section{Competing interests}

The authors declare that they have no competing interests.

Received: 29 August 2019 Accepted: 15 October 2019

Published online: 30 October 2019

\section{References}

1. Brahmer JR, Rodriguez-Abreu D, Robinson AG, Hui R, Csoszi T, Fulop A, et al. Health-related quality-of-life results for pembrolizumab versus chemotherapy in advanced, PD-L1-positive NSCLC (KEYNOTE-024): a multicentre, international, randomised, open-label phase 3 trial. Lancet Oncol. 2017;18:1600-9.

2. O'Neil BH, Wallmark JM, Lorente D, Elez E, Raimbourg J, Gomez-Roca C, et al. Safety and antitumor activity of the anti-PD-1 antibody pembrolizumab in patients with advanced colorectal carcinoma. PLoS One. 2017;12:e0189848.

3. Bilgin B, Sendur MA, Bulent Akinci M, Sener Dede D, Yalcin B. Targeting the PD-1 pathway: a new hope for gastrointestinal cancers. Curr Med Res Opin. 2017;33:749-59.

4. Reck M, Rodriguez-Abreu D, Robinson AG, Hui R, Csoszi T, Fulop A, et al. Pembrolizumab versus chemotherapy for PD-L1-positive non-small-cell lung cancer. N Engl J Med. 2016:375:1823-33.

5. Brahmer JR, Lacchetti C, Schneider BJ, Atkins MB, Brassil KJ, Caterino JM, et al. Management of immune-related adverse events in patients treated with immune checkpoint inhibitor therapy: American Society of Clinical Oncology clinical practice guideline. J Clin Oncol. 2018;36:1714-68.

6. Guercio G, Tutino R, Falco N, Cocorullo G, Salamone G, Licari L, et al. Solitary metastasis from melanoma causing bowel perforation. Ann Ital Chir. 2015;86. ePub.

7. Berger A, Cellier C, Daniel C, Kron C, Riquet M, Barbier JP, et al. Small bowel metastases from primary carcinoma of the lung: clinical findings and outcome. Am J Gastroenterol. 1999;94:1884-7.

8. Garwood RA, Sawyer MD, Ledesma EJ, Foley E, Claridge JA. A case and review of bowel perforation secondary to metastatic lung cancer. Am Surg. 2005; $71: 110-6$

9. Yang CJ, Hwang JJ, Kang WY, Chong IW, Wang TH, Sheu CC, et al. Gastrointestinal metastasis of primary lung carcinoma: clinical presentations and outcome. Lung Cancer. 2006;54:319-23.

10. Francisco LM, Sage PT, Sharpe AH. The PD-1 pathway in tolerance and autoimmunity. Immunol Rev. 2010;236:219-42.

11. Pardoll DM. The blockade of immune checkpoints in cancer immunotherapy. Nat Rev Cancer. 2012;12:252-64.

\section{Publisher's Note}

Springer Nature remains neutral with regard to jurisdictional claims in published maps and institutional affiliations.

\section{Submit your manuscript to a SpringerOpen ${ }^{\circ}$ journal and benefit from:}

- Convenient online submission

- Rigorous peer review

- Open access: articles freely available online

- High visibility within the field

- Retaining the copyright to your article

Submit your next manuscript at $\boldsymbol{\nabla}$ springeropen.com 